Arbois during the summer months. The great man was among other things no mean artist-some of his portraits are in the Louvre-and the writer, who is an art historian, thinks that the powers of close observation which enabled him to paint were a vital factor in his scientific work.

The second article is by E S Lazowski, now of Chicago, and S Matulewicz, now of Kinshasa, Zaire, who were medical colleagues in Poland during the second world war. Why they have remained silent about their piece of deception for so long they do not say.

During the German occupation of Poland a fifth of the population was murdered and many others were condemned to forced labour in Germany. Meanwhile epidemic typhus broke out, and doctors were required to notify cases to the German authorities, who confirmed the diagnosis, on specimens of blood posted to them, by the Weil-Felix reaction. This test, based on a discovery made also in Poland in the first world war, depends on the fact that in typhus a high titre of $\mathrm{O}$ agglutinin is developed in the blood for a strain of Proteus named X19. This test is easily performed and highly specific, a titre of 50 or more being absolutely diagnostic.

A Polish labourer on leave from Germany to visit his family "was ready to do anything, including committing suicide, to escape the misery of slavery in Germany." The authors therefore proposed an experiment to him, to which he agreed. He was given a single intramuscular dose of Proteus OX19 suspension and developed an agglutinin titre of 500. A specimen of his blood was then posted to a German State laboratory and thence reported positive by telegram. This was presented to the local German authorities, whereupon the man "was officially released from his duties in Germany, and he and all his family were excluded from future detention. The Germans were afraid of transferring 'infected' lice during the incubation period." Fear of transmitting the disease to Germany itself was an important motive then and later.

The authors then realised that the artificial Weil-Felix reaction could be used as a form of defence against the policies of the German government of occupation. Under the guise of "protein stimulation therapy," several forms of which were in vogue at the time, they immunised "selected patients with any suggestion of symptoms to epidemic typhus," controlling the numbers in accordance with the seasonal incidence of the disease. "Soon the number of reported cases was sufficiently large to declare the area of our practice (about a dozen villages) an 'epidemic area' with relative freedom from oppression." This practice continued for two years and escaped detection even when "a medical detachment of the German army made an inspection of our 'epidemic area' on the basis of information supplied by a collaborator." The Germans evidently suspected that a Polish doctor would "use the blood from one typhus patient for many other reported cases." Mercifully the Germans, still fearing exposure to infected lice, examined none of the supposed patients in their homes but confined themselves to taking further blood specimens in their own laboratory, which, of course, were always found positive.

The brief account given does not explain why two further circumstances did not arouse suspicion. The agglutinin titre produced by a single injection is likely to have been fairly constant and never high, whereas the response to the disease itself is widely variable, the titre sometimes reaching many thousands. More significant still, presumably all these "patients" survived, whereas the mortality of typhus is considerable. Whether the Germans were simply obtuse or were somehow outwitted over this we are not told. Should not the authors consider publishing a fuller account of this unique piece of deception? This should be possible even though their records (if any) no longer exist. But at least it is fortunate that this interesting echo of a hideous past has not been entirely lost to knowledge.

${ }^{1}$ Hemphill, M-L, ASM News, 1977, 43, 298.
${ }^{2}$ Lazowski, E S, and Matulewicz, S, ASM News, 1977, 43, 300.

\section{Diagnostic tests in rheumatology}

The absence of specific and reliable diagnostic tests leaves rheumatology as one of the few remaining havens for clinicians. Care and time spent in history-taking and examination reap greater rewards than laboratory and even radiological investigations; laboratory tests interpreted inflexibly often lead to diagnostic disaster.

The overlap of symptom and disease complexes in several rheumatic diseases prevents rigid demarcation. For example, morning stiffness, particularly of more than 30 minutes' duration, is useful in separating rheumatoid arthritis from degenerative joint disease, but it also occurs in any inflammatory joint disease, in polymyalgia rheumatica, and in some presentations of giant cell arteritis. A synmetrical polyarthritis with a positive rheumatoid factor test is usuak'y rheumatoid arthritis, but it may be the presenting fexure of systemic lupus erythematosus (SLE) or progressiv: systemic sclerosis (PSS). Raynaud's phenomenon, scleroractyly, and oesophageal abnormalities occur not only in PS' but also in SLE and rheumatoid arthritis and in long-stalding uncomplicated Raynaud's phenomenon, which is itclf also a recognised entity. ${ }^{1}$ Proximal muscle weakness is common to polyarteritis nodosa, SLE, PSS, dermatmyositis or polymyositis, and even polymyalgia rheumatic: Current diagnostic tests should do no more than influence thrclinician's judgment; a reasonably definite clinical diagnosj can always be made, often based partly on the presence ordbsence of abnormalities in the renal, pulmonary, or neuroogical systems. That odd entity, "undiagnosed collagen vascular disease for trial of steroid therapy," should never ye seen again.

What are the tests availablednd how useful are they? A few are absolutely diagnostic, sich as the finding of intracellular monosodium urate or alcium pyrophosphate crystals in synovial fluid for the dugnosis of gout or pseudogout. Both conditions may presen: as an inflammatory polyarthritis with morning stiffness, ard for this reason an examination of synovial fluid is always worth while-as well as for its place in the exclusion of infection.

Immunological tests in inflammatory joint disease are of limited value. They depend greatly on the source of reagent and on the experience of the laboratory personnel. In general, commercial kit tests are less reliable than those using individually prepared antisera, but are far easier to set up. Quality control is also less easy to achieve than in biochemical tests. Rheumatoid (IgM) factor tests are in most demand, yet perhaps they are the most disappointing. Those based on latex particles are negative in some $40 \%$ of patients with rheumatoid arthritis and are positive in $5-10 \%$ of normal individuals; in $15 \%$ of those with diseases such as sarcoidosis, liver disease, syphilis, and other infections; in $25 \%$ of the population over 70 years $^{2}$; and in many other rheumatic 
diseases as well as in pulmonary fibrosis. Sheep cell agglutination tests are negative in a higher proportion of rheumatoid arthritics, but at least positive results are almost always confined to some rheumatic disease. Positive rheumatoid factor tests in rheumatoid arthritis tend to be associated with a worse prognosis. IgG rheumatoid factor and "hidden" rheumatoid factor may be found in seronegative rheumatoid arthritis, but their diagnostic value is outweighed by their technical difficulties. ${ }^{3}$

A finding of antinuclear antibody is confined almost entirely to serious connective tissue diseases. The test is positive in some $90^{\circ}$ " of cases of SLE and has largely replaced the LE cell test. In decreasing frequency it is also positive in Sjögren's syndrome, systemic sclerosis, rheumatoid arthritis, and dermatopolymyositis as well as in chronic active hepatitis and occasional normal individuals. Antibody to single-stranded denatured DNA is found in $90^{\circ}$ o, of patients wich SLE, in many other connective tissue diseases, and in chronic active hepatitis; antibody to native double-stranded DNA is found in $40-60^{\circ}$ of patients with active SLE but uncommonly in other diseases. ${ }^{3}$ The extractable nuclear antigen (ENA) test is a good example of how the use of a procedure to delineate a heterogeneous clinical entity, mixed connective tissue disease, ${ }^{4}$ can be misleading. On prolonged observation the prognosis of patients with positive results was found to conform to their clinical status-SLE, PSS, and so on."

What tests sjould be done in the investigation of inflammatory polyartiritis? Besides measurements of the haemoglobin and erythrocyte sedimentation rate a chest radiograph will help to exclude sarcoidosis and carcinoma of the bronchus as occult causes. A low or grossly raised plasma uric acid concentratinn is a useful finding-though gouty patients may have a nornal result. Moderately raised concentrations occur in individuals who are normal, obese, on diuretics, or have occult myelophliferative disease-as well as after the odd aspirin or two. $t$.negative rheumatoid factor test will further stimulate the que for the nail changes of psoriasis and the possibility of Croh's disease or colitis, Reiter's syndrome or atypical ankylosing siondylitis. A positive antinuclear antibody test should alert the Clnician to the possibility of SLE or some

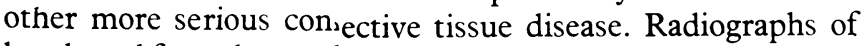
hands and feet taken early: $n$ the course of disease will be useful later to assess its progress.

${ }^{1}$ Stevens, M B, et al, New England $j$ urnal of Medicine, 1964, 270, 1218.

2 Litwin, R D, and Singer, J M, Arth'tis and Rheumatism, 1965, 8, 538.

${ }^{3}$ McDuffie, F C, and Bunch, T W, culletin on the Rheumatic Diseases, 1976-1977, 27, 900.

${ }^{4}$ Sharp, G C, et al, American fournal of Mudicine, 1972, 52, 148.

5 Brody, S, et al, Abstract, XIV International Congress of Rheumatology, San Francisco, June 1977.

\section{Surgical treatment of trigeminal neuralgia}

Though we still do not know what causes trigeminal neuralgia, some have argued that it is analogous to a localised form of sensory epilepsy. For this reason phenytoin ${ }^{1}$ and later carbamazepine were introduced into its treatment, with notable success $^{2}$ confirmed by clinical trials. ${ }^{34}$ Carbamazepine is effective within 48 hours; abolishes the pain or makes it easily tolerated in over $80 \%$ of patients; and, once the balance between therapeutic effect and side effects has been achieved, allows satisfactory maintenance therapy over many years in most patients.

The role of surgery for trigeminal neuralgia has therefore diminished, and most neurosurgeons now have much less experience in dealing with the condition. None the less destructive operations are still necessary in those patients in whom an adequate trial of carbamazepine has proved ineffective, and who have true idiopathic trigeminal neuralgia. The doctor must, of course, identify the rare cases in which trigeminal neuralgia is secondary to an underlying tumour compressing the trigeminal nerve or ganglion, or where it is symptomatic of a plaque of multiple sclerosis in the pons. An age of onset before 50, or other neurological symptoms or abnormal physical signs, are the main indications for investigating such patients.

High success rates have been claimed for various different surgical procedures. Sharr and Garfield ${ }^{5}$ have recently reviewed their experience in 81 patients of injection of the trigeminal ganglion or root with alcohol. In 71 patients followed up for an average of 3.3 years, 61 obtained complete symptomatic relief and six definite improvement, and in four the pain recurred. Relief of pain was not necessarily associated with total loss of cutaneous sensation. Previously Henderson ${ }^{6}$ had obtained total sensory loss in $87^{\circ}$ " of 196 injections, but one year after the procedure some recurrence of pain had occurred in $35^{\circ}{ }_{1}$. Injection of the ganglion or root carries no mortality rate, but keratitis due to denervation of the first division of the nerve, cranial nerve palsies due to misplaced injections, dysaesthesiae, and anaesthesia dolorosa (an intractable condition of pain despite evidence of total trigeminal block) are the most common complications encountered.

Other methods of destroying the pain-sensitive C fibres have been attempted by injecting phenol into Meckel's cave ${ }^{7}$ or more recently by radiofrequency thermocoagulation of the Gasserian ganglion and its posterior rootlets. ${ }^{8}{ }^{9}$ Satisfactory relief of pain was obtained in 121 of 135 patients, ${ }^{9}$ with preservation of the sense of touch, a distinct advantage, in $80 \%$ of patients. Electrocoagulation ${ }^{10}$ has also been used with less success, but the recurrence rate increases steadily in proportion to the duration of follow up.

For these reasons many workers have considered that a more permanent and complete ablation by fractional rhizotomy (intradural or extradural) is preferable to repeated injections of the ganglion. ${ }^{11-14}$ Complete relief of pain by this method is obtained in $95 \%$ of patients, and the recurrence rate in most series has been considerably smaller than that obtained by injection of the ganglion. There is an operative mortality rate of $1 \%$-while complications, including those affecting the eye $(10 \%)$, facial dysaesthesiae $(10-20 \%)$, and cranial nerve injury $(5-10 \%)$, occur significantly more often ${ }^{13}$ with trigeminal rhizotomy, and must be weighed carefully against the more complete and long-lasting benefits. The incidence of dysaesthesiae after rhizotomy may be lessened by a previous trigeminal injection.

Whatever procedure is used, certain principles apply. Surgical ablation for pain that is not trigeminal neuralgia gives disastrous results, so that accurate diagnosis is vital. The longer the follow-up, the higher the recurrence rate. Pain after rhizotomy which develops in a division unaffected at the time of surgery may be considered to be an extension of the disease rather than a failure of primary treatment; but it obviously requires a further procedure. ${ }^{6}$ Sparing of the first division of the nerve by a fractional section or injection reduces the incidence of keratitis. Other procedures (retrogasserian rhizotomy, neurolysis, and decompression of the ganglion) have enjoyed 